\title{
COVID-19 and Cancer Care in Indonesia: What We Have Done in Dharmais Cancer Center Hospital
}

\author{
Hendi Setiadi ${ }^{1}$, Fifi Dwijayanti ${ }^{2}$, Martya Rahmaniati Makful ${ }^{3}$ \\ ${ }^{1}$ Master Study Program of Biostatistics and Population, Faculty of Public Health, Universitas Indonesia, Depok, Indonesia \\ ${ }^{2}$ Research and Development Department, Dharmais Cancer Hospital - National Cancer Center, Jakarta, Indonesia \\ ${ }^{3}$ Department of Biostatistics and Population Studies, Faculty of Public Health, Universitas Indonesia, Depok, Indonesia
}

At the end of 2019, the novel coronavirus (CoV) of severe acute respiratory syndrome (SARS), named SARSCoV-2 was approved as a microbial agent that causes viral pneumonia in patients who are linked epidemiologically to the seafood market in Wuhan (Wuhan) Hubei province, China [1]. The World Health Organization (WHO) stated the coronavirus or COVID-19 as a pandemic because it has spread globally in the world since March 11, 2020 [2]. There have been more than 8 million cases reported with more than 450 thousand deaths around the world until June 19, 2020 [3]. Indonesia reported the first cases of COVID-19 in early March 2020 and currently 43,803 cases with 2,373 deaths [4]. Indonesia has the highest cases of COVID-19 in Southeast Asia [5].

WHO stated that according to the current evidence, coronavirus is transmitted among people through respiratory droplets and contact routes [6-8]. Droplet transmission is different from airborne disease. Droplet transmission occurs when a person closely contacted (within $1 \mathrm{~m}$ ) with someone who has respiratory symptoms (e.g. coughing or sneezing,) and was therefore at risk of having his/her mucosae (mouth and nose) or conjunctiva (eyes) exposed to potentially infective respiratory droplets. Droplet transmission may also occur through fomites in the immediate environment around the infected person [9]. Increasing cases occur continuously become alert for our institution as health care providers.

Based on the spreading of the virus and the high Case Fatality Rate (CFR), it showed how serious the disease. Dharmais Cancer Hospital is a national cancer referral hospital that treated patients with cancer who are immunocompromised. Cancer patients has a higher risk of being infected with coronavirus compared with non-cancer patients because of the myelosuppressive effect of treatment and their disease itself, which suppresses the immune system [10]. It is similar to CFR which seems to be highly variable but increases in patients with medical comorbidities and those who developed severe respiratory symptoms. In China, CFR in the non-cancer patient was suggested to be as high as $8 \%$ to $15 \%$, but higher on cancer patients. Patients with cancer have a higher risk and a longer time to recover their immunity especially if they undergo treatment such as chemotherapy, surgery, and radiotherapy [11]. Our institution tried to control and prevent the spreading of COVID-19 disease for patients and workers' safety.

No vaccine against COVID-19 is currently available. The vulnerability of hospitals to viruses spreading and healthcare workers infection, makes our institution gave the best efforts to prevent the disease. Dharmais Cancer Hospital makes efforts to prevent virus transmission virus by using Personal Protective Equipment (PPE) and manage the patients. The hospital management has changed the policy during the COVID-19 outbreak. All healthcare must use safety protocol for the prevention of infectious diseases. These efforts were done to maintain the safety of patients and healthcare workers. According to the Centers for Disease Control and Prevention $(C D C)$ recommendation, healthcare workers should use personal protective equipment and implement a standard, contact, and airborne precautions including the use of eye protection. Health care workers should wear gowns, gloves, and either an N95 respirator plus a face shield or goggles or a powered, air-purifying respirator [12].

Limiting the patient to reduce density in the hospital is carried out so they can keep physical distancing. Education and information are always provided through electronic and social media such as banners, TV shows, and broadcasts to remind everyone to do hygiene and sanitation. Dharmais also manage that one patient only comes with one caregiver, except the patient with "unwell" conditions and there are no visit hours for inpatient. The effort for limiting contact has done in all units including inpatient and outpatient care.

In inpatient care, all patients before undergoing treatment such as chemotherapy, radiation, or surgery are tested for COVID-19 test. Patients with positive results will be transferred to the negative pressure isolation room until the results show negative for two times swab tests. The Infection Prevention and Control Team (IPC / PPI) will track who has a history of contact with this patient. All people who have close contact 
will be given a rapid test to screen for virus transmission. Person Under Monitoring (ODP) will be monitored by the surveillance team for 14 days or until the patient was declared cured after testing for COVID-19.

In outpatient care, patient restrictions are carried out to reduce the number of people in the hospital so that they are not too crowd and can keep their physical distance. Rescheduling the visits of new patients, or post-response the second consultation for patients who already had treatment access in initial health care were being conducted. Making an appointment online is also being done to reduce patients to come into the hospital. Telemedicine also has an important part in our strategy to develop. In the counter unit administration, Dharmais put on a plastic barrier to prevent the spreading of the droplets between patients and healthcare workers. The administrative workers should use masks and gloves during work.

Dharmais also set out the patients who would come into the hospital for temperature screening and screening form checked. Screening of patients before come into hospital is divided into two namely firstly and secondary screening. The first screening teams are all employees who volunteering to be a team of COVID-19. The first screening task is checking the body temperature, patient's screening form, and asked for the purpose of coming into the hospital. It is intended that patients who enter have completed all administrative documents so that they can be orderly in receiving services. Patients with good condition will be given a green sticker as a sign that the patient is safe to be provided services. Whereas, patients with a body temperature of more than 37.5 degrees celsius or have symptoms of COVID-19 will be given a red sticker and directed to go to the secondary screening.

The secondary screening follows up the patients with a red sticker for clinical examination. The team in secondary screening are doctors and nurses. The secondary screening task is to examine the patient clinically. Patients will be examined for symptoms that their feels are COVID-19 symptoms or cancer symptoms. Patients who can enter the hospital will be given a green sticker without removing the red sticker, this shows that even doctors or nurses in the clinic must be careful in handling these patients.

COVID-19 outbreak has a big impact on many sectors, especially healthcare providers. Previously, some hospitals served treatment for cancer patients. But currently, they limiting it by referring the patients to Dharmais hospital. This situation impacts on the delay of patient diagnosis and treatment. All services should be done quickly to minimize contact between patients and medical workers for safety.
Dharmais has tried the best for limiting contact based on government recommendations. However, late diagnosis and treatment in cancer patients were very dangerous because it can reduce survival rates. Cancer will spread throughout the body when treatment is delay. Therefore, further research is needed regarding the impact of COVID-19 outbreaks on the survival rates of cancer patients. Clinical research related to the manifestations and prognostics of patient cancer with COVID-19 is also needed to increase survival rates.

Now, Indonesia is in a "New Normal Era" after passing large-scale social restrictions. As we know, COVID-19 cases are still high and even have increased until more than a thousand cases in the last few days. Some sectors are overcrowding such as markets and public transportation. Evaluation of this situation is needed because it gives the impact of the increase in the number of patients.

It is not easy to work in this situation. Medical teams are not the frontline in dealing with this situation, but they are the last guard when patients need treatment. Keeping healthy behavior and eating nutritious foods is the first step in preventing the disease and increase immunity. Honesty in providing information related to health conditions to medical workers is also important to help prevent disease. In some cases, medical teams died while working because of dishonesty patients about a history of contact so make the virus spread widely. Health workers who have tried to take care of patients died. Discipline is also very necessary to reduce case mortality by following the health protocol continuously. We believe Indonesia can face this situation if all communities are disciplined and aware of healthiness.

Corresponding author:

Hendi Setiadi

Master Study Program of Biostatistics and Population, Faculty of Public Health, Universitas Indonesia, Depok, Indonesia Email: hendisetiadi274@gmail.com

\section{REFERENCES}

1. Zhu N, Zhang D, Wang W, Li X, Yang B, Song J, et al. A novel coronavirus from patients with pneumonia in China, 2019. N Engl J Med. 2020;382(8):727-33.

2. World Health Organization. Coronavirus disease 2019 (COVID-19) Situation Report [Internet]. 2020 [cited 2020 Jun 19]. Available from: https://www.who.int/ emergencies/diseases/novel-coronavirus-2019/ situation-reports 
3. World Health Organization. Coronavirus disease (COVID-19) pandemic [Internet]. Coronavirus disease. 2020 [cited 2020 Jun 19]. Available from: https://www. who.int/emergencies/diseases/novel-coronavirus-2019

4. Worldmeter. Corona Virus in Indonesia [Internet]. 2020 [cited 2020 Jun 19]. Available from: https://www. worldometers.info/coronavirus/country/indonesia/

5. Worldometer. COVID-19 CORONAVIRUS PANDEMIC [Internet]. Coronavirus disease. 2020 [cited 2020 Jun 19]. Available from: https://www.worldometers.info/ coronavirus/

6. World Health Organization. Modes of transmission of virus causing COVID-19. Sci Br. 2020;(March):19-21.

7. Liu J, Liao X, Qian S, Yuan J, Wang F, Liu Y, et al. Community Transmission of Severe Acute Respiratory Syndrome Coronavirus 2, Shenzhen, China, 2020. Emerg Infect Dis. 2020;26(6):1320-3.

8. Chan JFW, Yuan S, Kok KH, To KKW, Chu H, Yang $J$, et al. A familial cluster of pneumonia associated with the 2019 novel coronavirus indicating person- to-person transmission: a study of a family cluster. Lancet [Internet]. 2020;395(10223):514-23. Available from: http://dx.doi.org/10.1016/S0140-6736(20)30154-9

9. Ong SWX, Tan YK, Chia PY, Lee TH, Ng OT, Wong MSY, et al. Air, Surface Environmental, and Personal Protective Equipment Contamination by Severe Acute Respiratory Syndrome Coronavirus 2 (SARS-CoV-2) from a Symptomatic Patient. JAMA - J Am Med Assoc. 2020;323(16):1610-2.

10. Patel R, Park J, Shah A, Saif MW. COVID-19 and Cancer Patients. Cancer Med J. 2020;3(1):40-8.

11. Rio C del, Malani PN. COVID-19-New Insights on a Rapidly Changing Epidemic. JAMA - J Am Med Assoc. 2020;323(11):1092-3.

12. Centers for Disease Control and Prevention (CDC). Strategies to Optimize the Supply of PPE and Equipment [Internet]. Healthcare professionals. 2020 [cited 2020 Jun 19]. Available from: https://www. cdc.gov/coronavirus/2019-ncov/hcp/ppe-strategy/ 